\title{
NEW INDONESIAN COUNTRY RECORDS AND SPECIES INFORMATION FOR MEALYBUGS (HEMIPTERA PSEUDOCOCCIDAE) IN WIRJATI'S HISTORIC COLLECTION $\left({ }^{1}\right)$
}

\author{
(*) School of Environmental and Natural Resource Sciences, Faculty of Science and Technology, National University of \\ Malaysia, Bangi 43600, Selangor, Malaysia. E-mails: dsartiami@yahoo.com and idrisyatie@yahoo.com.my \\ (**) Department of Plant Protection, Faculty of Agriculture, Bogor Agricultural Institute, Jl. Kamper Level 5 Wing 7 , \\ Darmaga, Bogor 16680, West Java, Indonesia. \\ (**) Plant Pest Diagnostic Center, California Department of Food and Agriculture, 3294 Meadowview Road, \\ Sacramento, California 95832, U.S.A. E-mail: gillian.watson@cdfa.ca.gov \\ $\left({ }^{\circ}\right.$ ) Malaysia Agricultural Research and Development Institute, Pesiaran MARDI-UPM, Serdang 43400, Selangor, \\ Malaysia.E-mail: roff@mardi.gov.my \\ (†) Corresponding author: dsartiami@yahoo.com
}

Sartiami D., Watson G.W., Mohamad Roff M.N., Idris A.B. - New Indonesian country records and species information for mealybugs (Hemiptera Pseudococcidae) in Wirjati's historic collection.

Between 1955 and 1960, a collection of slide-mounted mealybugs (Hemiptera: Coccomorpha: Pseudococcidae) was prepared by Mrs Wirjati, using samples collected between 1916 and 1960 from a variety of hosts in Indonesia. The collection is a record of which species were established in the country in 1960. The slides, deposited at the Indonesian Ministry of Agriculture in Java, are the earliest material in the national mealybug collection. There are 181 temporary slide mounts of unstained, waxy mealybugs mounted in agar or gum chloral media that have dried out to varying degrees. Specimens were retrieved from 50 representative slides and re-mounted as stained, archival mounts in Canada balsam. The method used to retrieve the specimens from the old mounts is described. The re-mounted specimens were re-identified using up-to-date, published identification keys, resulting in new identities for most of the specimens. Fifteen species belonging to 12 genera were identified, however specimens from four of the slides could not be identified beyond genus level. The Wirjati slides of Antonina thaiensis Takahashi, Hordeolicoccus nephelii (Takahashi) and Paraputo corbetti (Takahashi) are new country records. The differences between the fauna in Wirjati's collection and that found in Indonesia today are discussed.

KEY WoRDS: national collection, historic fauna, Wirjati, slide curation, re-mounting.

\section{INTRODUCTION}

The Indonesian national collection of mealybugs, made by Mrs Wirjati between 1950 and 1960, has been stored at the Indonesian Centre for Agricultural Biotechnology and Genetics Resources Research and Development (ICABGRRD), Indonesian Agency for Agricultural Research and Development, Ministry of Agriculture (Jalan Tentara Pelajar No. 3 Cimanggu, Bogor, West Java), since the 1950 s. It consists of 181 slide mounts stored in three wooden boxes. The significance of Wirjati's collection is that it records which species were established in Indonesia between 1916 and 1960, providing baseline information that makes it possible to recognize which of the mealybug species are likely to have become established in Indonesia since 1960.

In the Wirjati collection, the slide labels were written in pencil or ink and were attached using water-soluble glue. Often the data noted on labels is incomplete. The condition of the original slide mounts is very poor, with dirt and mould on the exterior surfaces, the mountant turned brown or crystallized, and sometimes with large air gaps beneath

\footnotetext{
${ }^{1}$ Original scientific contribution presented and discussed at XIV International Symposium on Scale Insect Studies, Catania-Italy, 13-16 June 2016.
}

the coverslips (Fig. I). In a few cases the specimens had been stained pink to purple using an unknown stain (Fig. II), but most of the specimens are unstained. In a single slide mount there are sometimes as many as seven specimens, not always all of the same species. It was possible to deduce that Wirjati's slides were made with water-soluble mountants because the edges of each coverslip had been sealed with oil-based paint to minimise evaporation of water from the mounts.

In each of the original mounts, evaporation of water through the imperfect seal has reduced the volume of the mountant so that the coverslip has been pulled down onto the specimens, crushing them. The two surfaces have become pressed together, making it extremely difficult to distinguish dorsal structures from those on the venter. The same phenomenon was noted in old slide mounts in the aphid collection at The Natural History Museum, London, UK (BROWN, 1997).

In some of the Wirjati mounts, the mountant (possibly an agar solution) was still liquid. In others, the mountant (possibly a gum chloral mixture related to Hoyer's Medium or Berlese Fluid) had dried out completely, leaving either paths of a clear, hard, glassy substance and large air spaces under the coverslip, or crystals attached to the insect cuticle (especially the body setae), or both. The variety of ways in which the mountants had behaved suggested that several different recipes had been used. In all cases, the insect 


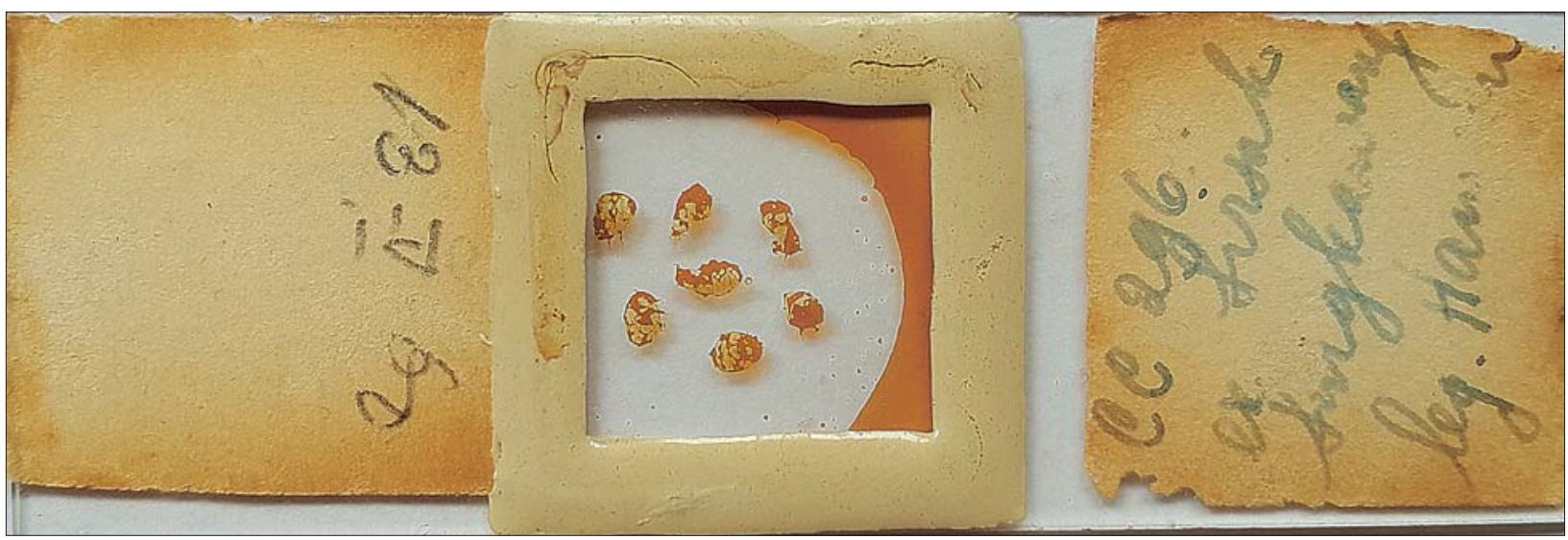

Fig. I - A Wirjati slide with air beneath the coverslip.

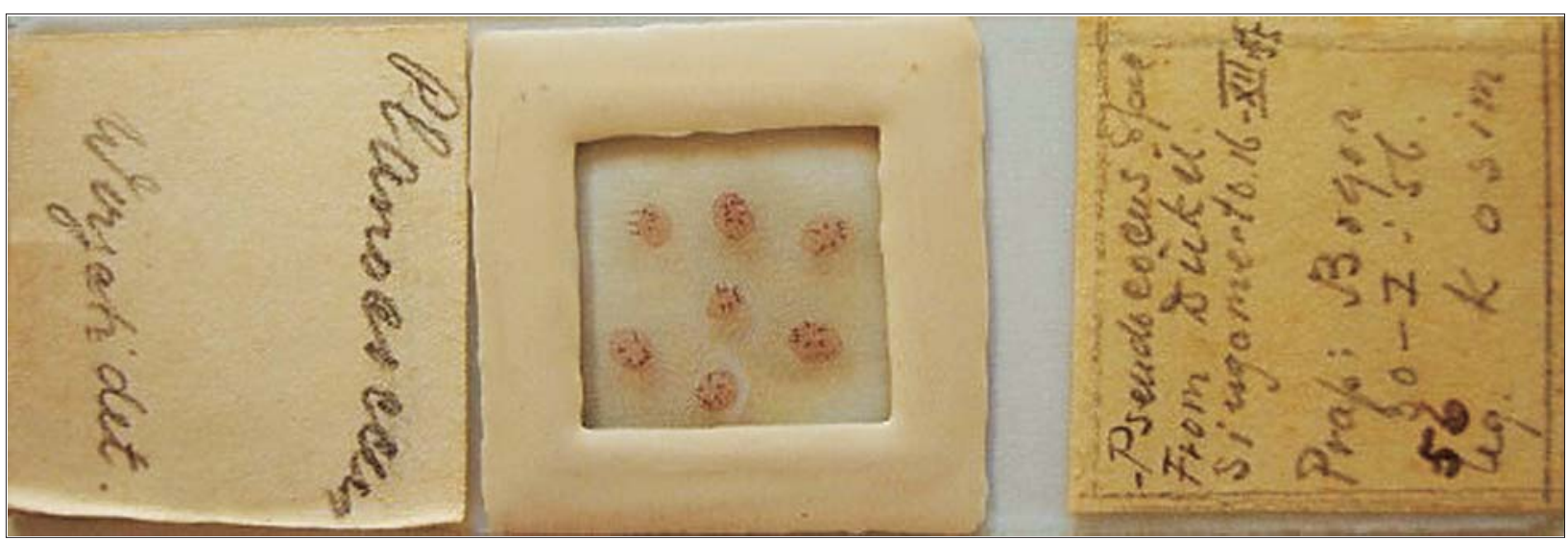

Fig. II - A Wirjati slide containing seven specimens stained pink to purple.

cuticles are still visible and the antennal segments can be counted at low power. However, it is difficult or impossible to see the setae either in the cerarii or on the body, and smaller structures like trilocular pores are not visible. Often the cuticles are badly crumpled and many of the cerarian setae have been lost. In a few of the mounts some of the more distinctive species can be recognized (for example, a few specimens of Antonina sp., Exallomochlus hispidus (Morrison), Dysmicoccus lepelleyi (Betrem) and Hordeolicoccus nephelii (Takahashi)), but in most of the original mounts the insects are not identifiable.

In order to re-identify the mealybugs to modern standards, the specimens needed to be removed from the old temporary mounts, stained and then re-mounted as permanent mounts in Canada balsam. This improved the quality of the preparations to a level where they could be accurately identified to species level and used as a permanent reference resource by entomologists.

The aim of this study was to re-mount a representative part of Wirjati's collection of mealybugs and re-identify the specimens, to record the species of mealybug present in Indonesia in the past. The resultant species records are compared with recent records from Indonesia.

\section{MATERIALS AND METHODS}

EXTRACTION OF SPECIMENS FROM OLD TEMPORARY SLIDE MOUNTS

Fifty old, temporary slide mounts from the Wirjati collection were selected for re-mounting. They were selected to represent samples from a variety of host-plants. The process of re-mounting is difficult and time-consuming, and only worth doing if the specimens are in good condition. Each slide was processed to extract the specimens as follows:

1. The slide was photographed in order to preserve the data in case the writing washed off during the remounting process, and to record the number of specimens in the mount.

2. To access the mountant, the paint seal was scratched and broken off the edge of the coverslip very carefully with a small, stiff spatula using horizontal movements, until all the coverslip edges had been exposed.

3. The tip of a mounted needle was used to scrape the last traces of the sealant from the coverslip edges, to completely expose the mountant in the crack. This ensured that the coverslip could lift off freely.

4. Under a stereomicroscope, a fine paintbrush was used to rinse the crack around the edge of the coverslip with $95 \%$ isopropanol, to remove any traces of paint or grease. Contact with the labels was avoided in case the alcohol smudged or dissolved anything written in ink.

5. The slide was placed in a petri dish, covered with cold water, and the dish was covered and labled with the date. The dish was placed on a cold, thermostatically controlled hotplate at room temperature and heated to $60{ }^{\circ} \mathrm{C}$.

6. After a short time the water-soluble glue dissolved and the labels floated off the slide. The glue was gently 
rinsed off their backs before they were removed from the dish and placed face down on the glass lid to dry.

7. Heating continued and the slide was checked under the stereomicroscope periodically to monitor progress of dissolution of the mountant. In very thin mounts, this process was very slow, so a bulb pipette was used to gently flush water beneath the coverslip to remove the mountant faster. Only when the coverslip moved when shaking the dish slightly was the slide ready for the next step.

8. Under the stereomicroscope, a mounted needle was used to gently push the coverslip sideways. If it resisted movement at all, the heating and soaking process was resumed. Another attempt would be made after some time; once the coverslip floated off the specimens easily, their positions were noted.

9. If the specimens were still stuck on the slide, they were soaked until they floated off into the water. If they were attached to the coverslip, it was turned over so that the specimens were on the upper surface, and soaking continued until they floated off. A very gentle nudge with a spatula or paintbrush to encourage them to move did not hurt them, but no attempt was made to force them off the glass using instruments as this would have damaged them.

10. Once the speciments were floating free in the water (or on the surface), a spatula was used to lift and transfer them into clean water in a cavity block, where they were covered and soaked until all traces of mountant inside them had disappeared. At this stage they were ready to be processed into a new slide mount.

PREPARATION OF RETRIEVED SPECIMENS INTO PERMANENT SLIDE MOUNTS

The slide preparation method given by SIRISENA et al. (2013) was used to re-mount the specimens. The process involved maceration (if necessary), staining, dehydration, de-waxing, clearing and mounting.

First, if the specimens contained body contents they were macerated by gently heating in $10 \% \mathrm{KOH}$. Any embryos or eggs still inside the body could sometimes be gently extracted through an existing hole in the cuticle, or a small hole could be made in the cuticle if necessary to remove them; however if these fragments resisted removal then they were left to avoid causing serious damage to the insect cuticle. If the body had been folded or crumpled, sometimes it could be gently re-shapped while in $\mathrm{KOH}$; however this can only be done with specimens retrieved from aqueous mountants. Specimens retrieved from Canada balsam mounts are far too stiff, brittle and fragile to attempt dissection or re-shaping.

After maceration, the specimens were soaked in water to rinse out the $\mathrm{KOH}$. If the body was mis-shapen, sometimes it could be gently re-shaped while in water. Subsequently any remaining $\mathrm{KOH}$ in the specimens was neutralised in acidified $80 \%$ isopropanol before the cuticle was stained with acid fuchsin. The specimens were then briefly rinsed in acidified $80 \%$ isopropanol and then dehydrated in $95 \%$ isopropanol, before being cleared in clove oil and then mounted in Canada balsam. The slides were placed in a thermostatically controlled oven at $40{ }^{\circ} \mathrm{C}$ to dry.

After three months, the slides were ready for labels to be attached using non-water-soluble glue. If only one new slide had been made, the original labels were attached to the new mount. However, if more than one slide was made, then the original labels were attached to the best slide mount and the other slides were labeled with replica labels printed from the photograph of the original slide. The re-mounted specimens were identified using keys in WiLLIAMs (2004) and COX (1989).

Re-mounting and re-identification were carried out at the Plant Pest Diagnostic Center, California Department of Food and Agriculture, Sacramento, California, U.S.A. (PDDC) and the Department of Plant Protection, Faculty of Agriculture, Bogor Agricultural University, Bogor, Indonesia.

\section{RESULTS AND DISCUSSION}

Fifty original slides were re-mounted and re-identified. Some crumpled specimens resisted being re-shaped; this made the recognition of morphological characters and identification to species level more difficult.

Of seven slides that were undetermined by Wirjati, six were identified to species and the remaining one was identified as Monophlebidae sp. One sample, identified by Wirjati as "Diaspididae", contained Odonaspis serrata BenDov (Diaspididae) and the mealybug Chaetococcus bambusae (Maskell). Of four slides determined by Wirjati only to family Pseudococcidae, three were re-identified to species level and one could only be re-identified to genus Pseudococcus. Of 23 slides determined by Wirjati to genus level, i.e. Antonina, Dysmicoccus, Nipaecoccus and Pseudococcus, four were re-identified to genus level and the remaining 19 to species level. Of 15 samples determined by Wirjati to species level, the identity of four were confirmed in the re-mounted material and the remaining 15 slides proved to belong to different genera and/or species than the original determination. The original determinations of the 50 Wirjati slides and their re-identifications are given in Table 1. Below, each of the mealybug species is discussed, and the species represented in the Wirjati collection are compared to the mealybug species present in Indonesia today.

\section{Antonina thaiensis Takahashi}

This is the first record of $A$. thaiensis from Indonesia, collected on bamboo in Java, Bogor, 17.xii.1957 by Soehardjan. WiLliams (2004) did not include Indonesia in the distribution of this species.

\section{Chaetococcus bambusae (Maskell)}

Chaetococcus bambusae was found among specimens of Odonaspis serrata Ben-Dov (Diaspididae) on one Wirjati slide, which she had determined as Diaspididae. This mealybug had been recorded from Indonesia previously; the earliest record was from Java, collected by E. Jacobson on bamboo in 1907 (WILLIAMS, 2004).

\section{Dysmicoccus brevipes (Cockerell)}

The earliest record of this species from Indonesia was a plant quarantine interception at Washington DC, USA, from Java, Bogor, in 1917. The 1960 sample in Wirjati's collection is additional evidence of its occurrence in Indonesia (West Java, Subang, Pusakanegara).

\section{Dysmicoccus lepelleyi (Betrem)}

Betrem described $D$. lepelleyi from a specimen sent to him by R.H. Le Pelley in 1937, collected on Annona muricata (Annonaceae) from Java, Bogor. The earliest Indonesian collection was made by van der Goot in 1928, also from Java, Bogor, on Mangifera indica (Anacardiaceae) (WILliAms, 2004). Wirjati's 1949 sample from 
Table 1 - List of original determinations and re-identifications of specimens remounted from Wirjati's slides.

\begin{tabular}{|c|c|c|c|c|c|}
\hline No. & Slide code & Original determination & Collection data & Present identification & Comments \\
\hline 1 & $\mathrm{CC} 162$ & Undetermined & $\begin{array}{l}\text { Bogor, 18.vii.1957, leg. } \\
\text { Wirjati, on Scombortia sp. }\end{array}$ & $\begin{array}{l}\text { Planococcus minor } \\
\text { (Maskell) }\end{array}$ & \\
\hline 2 & CC209 & Undetermined & $\begin{array}{l}\text { Sindangsari, 21.xi.1957, leg. } \\
\text { Hamann, on Manihot sp. }\end{array}$ & $\begin{array}{l}\text { Planococcus lilacinus } \\
\text { (Cockerell) }\end{array}$ & \\
\hline 3 & $\mathrm{CC} 235$ & Undetermined & $\begin{array}{l}\text { Bogor, xii.1957, leg. Tjoa Tjien } \\
\text { Mo, on Dendrobium sp. roots }\end{array}$ & $\begin{array}{l}\text { Pseudococcus cryptus } \\
\text { Hempel }\end{array}$ & $\begin{array}{l}\text { Earliest } \\
\text { record }\end{array}$ \\
\hline 4 & CC280 & Undetermined & $\begin{array}{l}\text { Pusakanegara, 14.iii.1960, leg. } \\
\text { Dandi, on Glycine max }\end{array}$ & $\begin{array}{l}\text { Dysmicoccus brevipes } \\
\text { (Cockerell) }\end{array}$ & \\
\hline 5 & CC293 & Undetermined & $\begin{array}{l}\text { Lembang, Kalimantan, } \\
\text { 8.ix.1960, leg. Hamann, on } \\
\text { Capsicum sp. }\end{array}$ & $\begin{array}{l}\text { Planococcus minor } \\
\text { (Maskell) }\end{array}$ & \\
\hline 6 & CC295 & Undetermined & $\begin{array}{l}15 \mathrm{~km} \text { near Pontianak, } \\
\text { 3.v.1960, leg. Hamann, on } \\
\text { Mangifera indica }\end{array}$ & Monophlebidae & \\
\hline 7 & CC296 & Undetermined & $\begin{array}{l}\text { Singkawang, 13.v.1960, leg. } \\
\text { Hamann, on Annona muricata }\end{array}$ & $\begin{array}{l}\text { Planococcus minor } \\
\text { (Maskell) }\end{array}$ & \\
\hline 8 & $\mathrm{CC} 74$ & Diaspididae and 'spec 2' & $\begin{array}{l}\text {-, 24.v.1955, leg. Hamann, on } \\
\text { bamboo }\end{array}$ & $\begin{array}{l}\text { Chaetococcus } \\
\text { bambusae (Maskell), } \\
\text { Odonaspis serrata } \\
\text { Ben-Dov }\end{array}$ & \\
\hline 9 & $\mathrm{CC} 224$ & Pseudococcidae & $\begin{array}{l}\text { Bogor, 17.xii, 1957, leg. } \\
\text { Wirjati, on Crotalaria sp. }\end{array}$ & $\begin{array}{l}\text { Planococcus minor } \\
\text { (Maskell) }\end{array}$ & \\
\hline 10 & CC248 & Pseudococcidae & $\begin{array}{l}\text { Sukabumi, 25.viii.1958, leg. } \\
\text { Wirjati, on Mangifera indica }\end{array}$ & $\begin{array}{l}\text { Exallomochlus hispidus } \\
\text { (Morrison) }\end{array}$ & \\
\hline 11 & $\mathrm{CC} 265$ & Pseudococcidae & $\begin{array}{l}\text { Bogor, 26.i.1959, leg. Wirjati, } \\
\text { on Solanum sanitwongsei }\end{array}$ & $\begin{array}{l}\text { Planococcus minor } \\
\text { (Maskell) }\end{array}$ & \\
\hline 12 & $\mathrm{CC} 270$ & Pseudococcus sp. & $\begin{array}{l}\text { Bogor, 13.x.1959, leg. Oey } \\
\text { Hong Peng, on Theobroma } \\
\text { cacao stem }\end{array}$ & $\begin{array}{l}\text { Exallomochlus hispidus } \\
\text { (Morrison) }\end{array}$ & \\
\hline 13 & $\mathrm{CC} 270$ & Pseudococcus sp. & $\begin{array}{l}\text { Bogor, 13.x.1959, leg. Oey } \\
\text { Hong Peng, on Theobroma } \\
\text { cacao stem }\end{array}$ & $\begin{array}{l}\text { Exallomochlus hispidus } \\
\text { (Morrison) }\end{array}$ & \\
\hline 14 & $\mathrm{CC} 270$ & Pseudococcus sp. & $\begin{array}{l}\text { Bogor, 13.x.1959, leg. Oey } \\
\text { Hong Peng, on Theobroma } \\
\text { cacao stem }\end{array}$ & $\begin{array}{l}\text { Exallomochlus hispidus } \\
\text { (Morrison) adult and } \\
\text { immature }\end{array}$ & \\
\hline 15 & CC287 & Pseudococcidae & Bogor, 5.iv.1960, - , on orchids & Pseudococcus sp. & \\
\hline 16 & $\mathrm{CC} 225$ & Antonina sp. & $\begin{array}{l}\text { Bogor, 17.xii.1957, on } \\
\text { Bambusa sp. }\end{array}$ & $\begin{array}{l}\text { Antonina thaiensis } \\
\text { Takahashi }\end{array}$ & $\begin{array}{l}\text { New country } \\
\text { record }\end{array}$ \\
\hline 17 & CC11 & Dysmicoccus sp. & $\begin{array}{l}\text { Bandung, 6.x.1955, leg } \\
\text { Hamann, on Annona squamosa }\end{array}$ & $\begin{array}{l}\text { Saisettia coffeae } \\
\text { (Walker) }\end{array}$ & \\
\hline 18 & $\mathrm{CC} 11$ & Dysmicoccus sp. & $\begin{array}{l}\text { Bandung, 6.x.1955, leg } \\
\text { Hamann, on Annona squamosa }\end{array}$ & $\begin{array}{l}\text { Dysmicoccus lepelleyi } \\
\text { (Betrem) }\end{array}$ & \\
\hline 19 & 179 & Nipaecoccus? & $\begin{array}{l}\text { Bogor, 20.vii.1957, leg Wirjati, } \\
\text { on Polyrachis sp. nest on palm }\end{array}$ & $\begin{array}{l}\text { Dysmicoccus lepelleyi } \\
\text { (Betrem) }\end{array}$ & \\
\hline 20 & 179 & Nipaecoccus? & $\begin{array}{l}\text { Bogor, 20.vii.1957, leg Wirjati, } \\
\text { on Polyrachis sp. nest on palm }\end{array}$ & Pseudococcus sp. & \\
\hline 21 & CC210 & Nipaecoccus sp. & $\begin{array}{l}\text { Sumatera, 26.XI.1957, leg. } \\
\text { Hamann, on virgin wood } \\
\text { (timber) }\end{array}$ & $\begin{array}{l}\text { Nipaecoccus nipae } \\
\text { (Maskell) }\end{array}$ & $\begin{array}{l}\text { Earliest } \\
\text { record }\end{array}$ \\
\hline 22 & CC152 & Planococcus sp. & $\begin{array}{l}\text { Bogor, }-, \text { leg. -, on Elettaria } \\
\text { cardamomum }\end{array}$ & Planococcus sp. & \\
\hline 23 & 17 & Pseudococcus sp. & No data & $\begin{array}{l}\text { Dysmicoccus lepelleyi } \\
\text { (Betrem) }\end{array}$ & \\
\hline 24 & $\mathrm{CC} 10$ & Pseudococcus sp. & $\begin{array}{l}\text { Bandung, 6.X.1956, leg } \\
\text { Hamann, on Psidium guajava }\end{array}$ & $\begin{array}{l}\text { Exallomochlus hispidus } \\
\text { (Morrison) adult and } \\
\text { immature }\end{array}$ & \\
\hline 25 & CC86 & Pseudococcus sp. & $\begin{array}{l}\text { Wanatjipta, 4.II.1956, leg. } \\
\text { Kosim, on Acalypha indica }\end{array}$ & $\begin{array}{l}\text { Planococcus minor } \\
\text { (Maskell) }\end{array}$ & \\
\hline 26 & CC91 & Pseudococcus sp. & $\begin{array}{l}\text { Perigi, 23.XII.1955, leg. } \\
\text { Kosim, on Pterocarpus indicus }\end{array}$ & Monophlebidae & \\
\hline 27 & CC195 & Pseudococcus sp. & $\begin{array}{l}\text { Bogor, 10.VIII.1957, leg. } \\
\text { Hamann, Hura polyanda }\end{array}$ & Planococcus sp. & \\
\hline 28 & $\mathrm{CC} 213 \mathrm{~b}$ & Pseudococcus sp. & $\begin{array}{l}\text { Sindangsari, 21.XI.1957, leg. } \\
\text { Hamann, on Coffea sp. }\end{array}$ & $\begin{array}{l}\text { Planococcus lilacinus } \\
\text { (Cockerell) }\end{array}$ & \\
\hline
\end{tabular}


Continued Table 1

\begin{tabular}{|c|c|c|c|c|c|}
\hline No. & Slide code & Original determination & Collection data & Present identification & Comments \\
\hline 29 & CC232 & Pseudococcus sp. & $\begin{array}{l}\text { Bogor, 1.i.1958, leg. Tjoa Tjien } \\
\text { Mo, on Vanda sp. }\end{array}$ & $\begin{array}{l}\text { Coccidae sp. } \\
\text { Dysmicoccus orchidum } \\
\text { Williams }\end{array}$ & \\
\hline 30 & CC236 & Pseudococcus sp. & $\begin{array}{l}\text { Bogor, 12.xii.1957, leg. Tjoa } \\
\text { Tjien Mo, on Vanda sp. }\end{array}$ & $\begin{array}{l}\text { Planococcus lilacinus } \\
\text { (Cockerell) }\end{array}$ & \\
\hline 31 & $\mathrm{CC} 12$ & $\begin{array}{l}\text { Anisococcus crawii (Coq)./ } \\
\text { Pseudococcus sp. }\end{array}$ & $\begin{array}{l}\text { Bandung, 6.x.1955, leg } \\
\text { Hamann, on Annona muricata }\end{array}$ & $\begin{array}{l}\text { Dysmicoccus lepelleyi } \\
\text { (Betrem) }\end{array}$ & \\
\hline 32 & C54 & $\begin{array}{l}\text { Pseudococcus sp. Re-identified } \\
\text { by Wirjati as Phenacoccus? }\end{array}$ & $\begin{array}{l}\text { Bogor, 26.xi.1955, leg. } \\
\text { Hamann, on Nephelium } \\
\text { lappaceum }\end{array}$ & $\begin{array}{l}\text { Exallomochlus hispidus } \\
\text { (Morrison) }\end{array}$ & \\
\hline 33 & CC56A & $\begin{array}{l}\text { Pseudococcus sp. Re-identified } \\
\text { by Wirjati as Phenacoccus }\end{array}$ & $\begin{array}{l}\text { Singomerto, 16.xii.1955, } \\
\text { leg. Kosim, on Lansium } \\
\text { domesticum }\end{array}$ & $\begin{array}{l}\text { Exallomochlus hispidus } \\
\text { (Morrison) }\end{array}$ & \\
\hline 34 & CC56/56A & $\begin{array}{l}\text { Pseudococcus sp. Re-identified } \\
\text { by Wirjati as Phenacoccus }\end{array}$ & $\begin{array}{l}\text { Singomerto, 16.xii.1955, } \\
\text { leg. Kosim, on Lansium } \\
\text { domesticum }\end{array}$ & $\begin{array}{l}\text { Exallomochlus hispidus } \\
\text { (Morrison) }\end{array}$ & \\
\hline 35 & CC114 & $\begin{array}{l}\text { Pseudococcus sp. Re-identified } \\
\text { by Wirjati as Phenacoccus }\end{array}$ & $\begin{array}{l}\text { Bogor, 5.iv.1958, leg. Hamann, } \\
\text { on Cynometra cauliflora }\end{array}$ & $\begin{array}{l}\text { Hordeolicoccus } \\
\text { nephelii (Takahashi) }\end{array}$ & $\begin{array}{l}\text { New country } \\
\text { record }\end{array}$ \\
\hline 36 & $\mathrm{CC} 24$ & Dysmicoccus obesus (Lobdell) & $\begin{array}{l}\text { Bogor, 23.viii.1955, leg. } \\
\text { Hamann, on Theobroma cacao }\end{array}$ & $\begin{array}{l}\text { Exallomochlus hispidus } \\
\text { (Morrison) }\end{array}$ & \\
\hline 37 & CC62 & Dysmicoccus obesus (Lobdell) & $\begin{array}{l}\text { Singomerto, 16.xii.1955, } \\
\text { leg. Kosim, on Cynometra } \\
\text { cauliflora }\end{array}$ & $\begin{array}{l}\text { Exallomochlus hispidus } \\
\text { (Morrison) }\end{array}$ & \\
\hline 38 & $\mathrm{CC} 42$ & Ferrissiana virgata & $\begin{array}{l}\text { Bogor, 10.x.1955, leg. Kosim, } \\
\text { on Dysoxylum macrocarpum }\end{array}$ & $\begin{array}{l}\text { Planococcus minor } \\
\text { (Maskell) }\end{array}$ & \\
\hline 39 & $1271 / 1$ & Formicoccus mangiferae Betrem &,,-- leg. Voss & $\begin{array}{l}\text { Paraputo corbetti } \\
\text { (Takahashi) }\end{array}$ & $\begin{array}{l}\text { New country } \\
\text { record }\end{array}$ \\
\hline 40 & 201 & $\begin{array}{l}\text { Planococcus (Pseudococcus) } \\
\text { citri }\end{array}$ & $\begin{array}{l}\text { Bogor, } 25 . X .1957, \text { leg. Wirjati, } \\
\text { on Theobroma cacao }\end{array}$ & $\begin{array}{l}\text { Planococcus minor } \\
\text { (Maskell) and one } \\
\text { coccinellid larva }\end{array}$ & \\
\hline 41 & CC250 & Planococcus kenyae Le Pelley & $\begin{array}{l}\text { Sukabumi, 25.viii.1958, leg. } \\
\text { Wirjati, on Prunella }\end{array}$ & $\begin{array}{l}\text { Planococcus minor } \\
\text { (Maskell) }\end{array}$ & \\
\hline 42 & $\mathrm{CC} 212 \mathrm{~b}$ & Pseudococcus debregeasiae & $\begin{array}{l}\text { Bogor, 17.i.1950, leg. Vos, on } \\
\text { Solanum sp. }\end{array}$ & $\begin{array}{l}\text { Planococcus lilacinus } \\
\text { (Cockerell) }\end{array}$ & \\
\hline 43 & CCV2006 & $\begin{array}{l}\text { Pseudococcus jacobsoni Green } \\
\text { det. Morrison }\end{array}$ & $\begin{array}{l}\text { Bogor, 17.i.1950, leg. Vos, on } \\
\text { Lansium domesticum }\end{array}$ & $\begin{array}{l}\text { Exallomochlus hispidus } \\
\text { (Morrison) }\end{array}$ & \\
\hline 44 & CCV2006/2006 & $\begin{array}{l}\text { Pseudococcus jacobsoni Green } \\
\text { det. Morrison }\end{array}$ & $\begin{array}{l}\text { Bogor, 17.i.1950, leg. Vos, on } \\
\text { Lansium domesticum }\end{array}$ & $\begin{array}{l}\text { Exallomochlus hispidus } \\
\text { (Morrison) }\end{array}$ & \\
\hline 45 & CCV2007 & Pseudococcus lepelleyi & $\begin{array}{l}\text { Bogor, xi.1949, leg. Vos, on } \\
\text { Musa sp. }\end{array}$ & $\begin{array}{l}\text { Dysmicoccus lepelleyi } \\
\text { (Betrem) }\end{array}$ & \\
\hline 46 & CC282 & Saccharicoccus sacchari & $\begin{array}{l}\text { Bogor, 26.iii.1960, leg. Wirjati, } \\
\text { on Saccharum officinarum }\end{array}$ & $\begin{array}{l}\text { Saccharicoccus } \\
\text { sacchari (Cockerell) }\end{array}$ & $\begin{array}{l}\text { Earliest } \\
\text { record }\end{array}$ \\
\hline 47 & CCV36 & $\begin{array}{l}\text { Pseudococcus tabayanus }= \\
\text { Pseudococcus crotonis }= \\
\text { Planococcus lilacinus }\end{array}$ & $\begin{array}{l}\text { Bogor, } 1927 \text {, leg. -, on } \\
\text { Theobroma cacao }\end{array}$ & $\begin{array}{l}\text { Exallomochlus hispidus } \\
\text { (Morrison) }\end{array}$ & \\
\hline 48 & CCV988.1 & $\begin{array}{l}\text { Phenacoccus ornatus Green det. } \\
\text { P. van der Goot }\end{array}$ & $\begin{array}{l}\text { Bogor, 27.v.1935, leg. Frassen, } \\
\text { on Psidium guajava }\end{array}$ & $\begin{array}{l}\text { Rastrococcus } \\
\text { chinensis } \text { Ferris } \\
\text { Planococcus minor } \\
\text { (Maskell) }\end{array}$ & $\begin{array}{l}\text { Earliest } \\
\text { record }\end{array}$ \\
\hline 49 & CC490 & Pseudococcus citri Risso & $\begin{array}{l}\text { Nganjuk, v.1916, leg. Rappard, } \\
\text { on Tectona grandis }\end{array}$ & $\begin{array}{l}\text { Maconellicoccus } \\
\text { hirsutus (Green) }\end{array}$ & $\begin{array}{l}\text { Earliest } \\
\text { record }\end{array}$ \\
\hline 50 & CCV513 & Pseudococcus crotonis & $\begin{array}{l}\text { Bogor, vii. 1927, leg. Vos, on } \\
\text { Theobroma cacao }\end{array}$ & $\begin{array}{l}\text { Exallomochlus hispidus } \\
\text { (Morrison) }\end{array}$ & $\begin{array}{l}\text { Earliest } \\
\text { record }\end{array}$ \\
\hline
\end{tabular}

Java, Bogor, is additional evidence; the species is probably endemic to Java.

Dysmicoccus orchidum Williams

Dysmicoccus orchidum is specific to orchid hosts. Williams (2004) mentioned that Franssen collected this species from Java, Bogor, on Phalaenopsis sp. in June 1935, which is the earliest record from Indonesia. Two morphological characters of this species evident in Wirjati's 1958 sample are the round-to-quadrate circulus not divided by an intersegmental line, and that the anal lobe cerarius sclerotization is paler near the bases of the conical cerarian setae.

Exallomochlus hispidus (Morrison)

This species is the commonest in the remounted slides from Wirjati's collection; out of 50 re-mounted slides, 14 contained E. hispidus. In 1958, Wirjati described the species as new under the name Pseudococcus dorsospinosus (WIRJATI, 1958); WiLLIAMS (2004) placed this name as a junior synonym of $E$. hispidus. Based on the specimen data 
in WirJati (1958) and Williams (2004), the sample prepared by Wirjati, collected in July 1927 by Vos, is the earliest record of $E$. hispidus from Indonesia.

\section{Hordeolicoccus nephelii Takahashi}

Wirjati's slide is the first record of $H$. nephelii from Indonesia. She had labeled the slide as Phenacoccus sp., collected by Hamann in Bogor, 5.iv.1958, on Nam-nam (Cynometra cauliflora, Fabaceae), a new host record for this species according to the host list in GARCÍA MORALES et al., 2016.

\section{Maconellicoccus hirsutus (Green)}

Wirjati's slide of $M$. histutus was prepared from a sample from Rappard's field work in East Java dated 1916, and was originally misidentified as Planococcus citri (Risso). The slide pre-dates the material listed in BETREM (1937), so it is the earliest record of $M$. hirsutus from Indonesia. The species is probably native to southern Asia (WILLIAMS, 2004).

\section{Nipaecoccus nipae (Maskell)}

The Wirjati slide of $N$. nipae, dated 26.x.1957, is the earliest record of this species from Indonesia. It was also collected by G.W. Watson from a palm in West Java, near Bandung, on 17.iv.1997 and 25.iv.1997 (WILLIAMS, 2004).

\section{Paraputo corbetti Takahashi}

Wirjati identified her single specimen as Formicoccus mangiferae Betrem (now Paraputo mangiferae (Betrem)); no locality or date were given. When the slide was remounted, re-identification indicated that the specimen is $P a$. corbetti. However, Williams (2004) concluded that Pa. corbetti is a variant of $P a$. mangiferae Betrem, 1937. Further study of additional samples from East Java, especially from mango, would help clarify whether $\mathrm{Pa}$. corbetti is a junior synonym of $P a$. mangiferae or a distinct species.

\section{Planococcus lilacinus (Cockerell)}

Planococcus lilacinus is the third commonest species in the re-mounted Wirjati slides, represented in eight slides out of 50. The earliest collection of Pl. lilacinus from Indonesia was by BETREM (1937) on the roots of coffee in Java, when he described the species under the name Pseudococcus deceptor. The placement of Ps. deceptor as a junior synonym of $\mathrm{Pl}$. lilacinus was made by Cox (1989).

\section{Planococcus minor (Maskell)}

Planococcus minor is the second commonest species in the remounted Wirjati slides, represented in 10 slides out of 50. Wirjati slide-mounted an alcohol sample collected by Frassen from Psidium guaja (Myrtaceae) in Java, Bogor, in 1935, and P. van der Goot identified it as Phenacoccus ornatus Green (now Rastrococcus ornatus (Green)). Remounting of this slide revealed two species, Pl. minor and Rastrococcus chinensis Ferris, indicating that there was a mixed population on the host. This is the earliest record of $P l$. minor from Indonesia.

One of Wirjati's slides had been misidentified as Planococcus kenyae Le Pelley, so re-identification of the sample as $P l$. minor confirms that $P l$. kenyae is not present in Indonesia. Planococcus kenyae is only found in the Afrotropical Region (CABI, 2016). The absence of $\mathrm{Pl}$. kenyae in Indonesia was supported by Cox (1989) and WiLLIAMS (2004). Records of the occurrence of this species in Indonesia in BETREM (1937) and KALSHOVEN (1981) need to be corrected.
Another of Wirjati's slides had been identified as Pl. citri (Risso) but re-mounting and re-identification revealed that it was $\mathrm{Pl}$. minor. The presence of $\mathrm{Pl}$. citri on Coffea sp. (Rubiaceae) in East Java was recorded by BETREM (1932), but WILLIAMS (2004) remarked that this species' population was less dominant than that of $\mathrm{Pl}$. minor. According to Williams \& Miller (2010), Pl. citri was listed from Krakatau Island on Macaranga tanarius (Euphorbiaceae) by DAMMERMAN (1948). Bearing in mind the difficulty of separating $P l$. citri from $P l$. minor based on morphology, the accuracy of these early records of $P l$. citri and the presence of this species in Indonesia are questionable.

\section{Pseudococcus cryptus Hempel}

One undetermined Wirjati slide contained a mealybug collected from Java, Bogor, on the root of Dendrobium sp. (Orchidaceae) in July 1957 by Tjoa Tjien Mo. The remounted specimen has been re-identified as Ps. cryptus and represents the earliest field-collected material of this species from Indonesia. WILLIAMS (2004) noted its occurrence in Indonesia from plant quarantine-intercepted material in USA but the date of that record was not given.

\section{Rastrococcus chinensis Ferris}

A Wirjati slide mount of a sample collected by Franssen from Java, Bogor, on Psidium guajava (Myrtaceae) on 27.v.1935 was identified by P. van der Goot as Phenacoccus ornatus Green (now R. ornatus). Nineteen years later, FERRIS (1954) collected mealybugs from China in 24.xii.1954 on an unknown host and described the species as Rastrococcus chinensis. The re-mounted Wirjati slide was found to contain a mixture of $R$. chinensis and $P l$. minor. The earliest Indonesian record of this species is from a collection by van der Goot at Java, Bogor, on Eugenia sp. (Myrtaceae) in 1928 (WILLIAMS, 1989).

\section{Saccharicoccus sacchari (Cockerell)}

Wirjati collected this specimen on Saccharum officinarum (Poaceae) in Java, Bogor, on 26.iii.1960. According to the material listed by WiLLIAMs (2004), this is the earliest record of Saccharicoccus sacchari from Indonesia. The distinctive hour-glass-shaped circulus in Wirjati's specimen can be recognized easily.

\section{INVASIVE MEALYBUG SPECIES IN INDONESIA}

Human trade in fresh plant material between countries facilitates the movement of mealybugs from one country to another; passive movement of first-instar nymphs (crawlers) or ovisac material containing eggs can also occur on the clothing of tourists (KAIRO et al., 2000). MANI \& SHIVAJU (2016) noted the spread of the invasive mealybugs Paracoccus marginatus Williams \& Granara de Willink and Phenacoccus manihoti Matile-Ferrero to other countries through the sale of planting material, plants and fresh fruits. At present, 98 species of mealybug have been recorded as present in Indonesia (García Morales et al., 2016). Some of these species are recognized as invasive in Indonesia, e.g. Phenacoccus solani Ferris and Pseudococcus jackbeardsleyi Gimpel \& Miller (WILLIAMS, 2004). Moreover, in recent years, several other new introductions of invasive mealybugs to Indonesia have been published: $P a$. marginatus (in MuNIAPPAN et al., 2009), and Ph. manihoti on Manihot esculenta (Euphorbiaceae) and Ph. solenopsis (Tinsley) on Solanum lycopersicum (Solanaceae) (in MuniaPPAn et al., 2011). Although SARTIAMI et al. (2010) published the discovery of Ps. jackbeardsleyi on Piper nigrum in Sukamulya, West Java, Indonesia, this species 
had been recorded previously from Indonesia by WILLIAMS (2004). None of these invasive species were found in Wirjati's collection, indicating that, when Wirjati prepared the slides in 1955-1960, they had not yet reached Indonesia.

\section{CONCLUSION}

Fifteen species of mealybugs were re-identified from Wirjati's collection, belonging to 12 genera: Antonina, Chaetococcus, Dysmicoccus, Exallomochlus, Hordeolicoccus, Maconellicoccus, Nipaecoccus, Paraputo, Planococcus, Pseudococcus, Rastrococcus and Saccharicoccus. Of the 50 slides re-mounted, four could only be identified to genus level. A few specimens of the families Monoplebidae, Coccidae and Diaspididae and a coccinellid larva were found among the mealybugs re-mounted from the collection. In the 50 Wirjati slides re-identified so far, were found the earliest field-collected Indonesian samples of six mealybug species: Exallomochlus hispidus, Maconellicoccus hirsutus, Nipaecoccus nipae, Planococcus minor, Pseudococcus cryptus and Saccharicoccus sacchari; also found were new country records of three species: Antonina thaiensis, Paracoccus corbetti and Hordeolicoccus nephelii. Based on the re-identified slides, none of the invasive species now found in Indonesia are represented in Wirjati's collection.

\section{ACKNOWLEDGEMENTS}

The authors thank Dr. Karden Mulya, Director of ICABGRRD, Ministry of Agriculture, Indonesia, for permission to re-mount and re-identify Wirjati's mealybug slides. We would also like to thank to Ms Susan McCarthy (Branch Chief, Plant Pest Diagnostic Center, California Department of Food \& Agriculture, Sacramento, U.S.A. (PDDC)), and Dr. Asih A. Nawangsih (Head of Department of Plant Pest and Diseases, Faculty of Agriculture, Bogor Agricultural University), Indonesia for permission to carry out re-mounting and re-identification of mealybugs at those facilities. We are grateful to Dr. Sri Suharni Siwi (ICABGRRD), for taking care of Wirjati's slide collection for many years, and to Dr. Soemartono Sosromarsono.(Bogor Agricultural University, Indonesia) and Dr. Martin Hauser (PPDC), for translating Betrem's papers.

\section{REFERENCES}

Betrem J.G., 1932 - Witte luis in Besoeki en Djember. Bergcultures, 6: 1178-1186.

Betrem J.G., 1937 - De morphologie en systematiek van enkele van de voornaamste witte-luizenso soorten van Java. - Archief Koffiecult. in Nederlandsch-Indie, 11: 1118.

Brown P.A., 1997 - A review of techniques used in preparation, curation and conservation of microscope slides at The Natural History Museum, London. - Biol. Curator, 10: 1-33.

CABI, 2016 - Planococcus kenyae (Coffee mealybug). At: www.cabi.org/isc/datasheet/41890 (accessed 17 August 2016).
Cox J.M., 1989 - The mealybug genus Planococcus (Homoptera: Pseudococcidae). - B. Brit. Museum (Nat. Hist.) Entomol., 58(1): 1-78.

DAMMERMAN K.W., 1948 - The fauna of Krakatau 18831933. K. Ned. Akad. van Wet., Afd. Natuur. (Tweede Sect.), 44: 1-594.

FERRIS, G.F., 1954 - Report upon scale insects collected in China (Homoptera: Coccoidea). Part V. (Contribution No. 89). - Microentomol., 19: 51-66.

García Morales M., Denno B.D., Miller D.R., Miller G.L., BEN-Dov Y., HARDY, N.B., 2016 - ScaleNet: a literature-based model of scale insect biology and sytematics. At: http://scalenet.info/scalesplace/ indonesia/Pseudococcidae/ (accessed 8 August 2016).

Kairo M.T.K., Pollard G.V., Peterkin D.D., Lopez V.F., 2000 - Biological control of the Hisbicus Mealybug Maconellicoccus hirsutus Green (Hemiptera: Pseudococcidae) in the Carribean. - Int. Pest Manage. Rev., 5: 241-254.

Kalshoven L.G.E., 1981 - Pests of Crops in Indonesia. Revised and translated by P.A. van der Laan. Ichtiar Baru. Van-Hoeve, Jakarta, Indonesia. 701pp.

Mani M., Shivaju C., 2016 - Mode of spread of mealybugs. In: Mealybugs and their Management in Agricultural and Horticultural Crops, Mani M. \& Shivaju C. Ed., Springer, New Delhi, pp. 113-116.

Muniappan R., Shepard B.M., Watson G.W., Carner G.R., Sartiami D., Rauf A., Hammig M.D., 2009 First Report of the papaya mealybug, Paracoccus marginatus (Hemiptera: Pseudococcidae), in Indonesia and India. - J. Agr. Urban Entomol., 25(1): 37-40.

Muniappan R., Shepard B.M., Watson G.W., Carner G.R., Rauf A., Sartiami D., Hidayat P., Afun J.V.K., Goergen G. \& Ziaur Rahman A.K.M., 2011 - New records of invasive insects (Hemiptera: Sternorrhyncha) in Southeast Asia and West Africa. - J. Agr. Urban Entomol., 26(4): 167-174.

Sartiami D., Sihombing E.R., Balfast R., 2010 Identifikasi species kutu putih (Hemiptera: Pseudococcidae) pada tanaman lada (Piper nigrum Linn.). In: Prosiding Seminar Nasional V: Pemberdayaan Keanekaragaman Serangga untuk Peningkatan Kesejahteraan Masyarakat. Perhimpunan Entomologi Indonesia, pp. 217-227.

Sirisena U.G.A.I., Watson G.W., Hemachandra K.S., WiJayagunaseKara H.N.P., 2013 - Preparation of Hemiptera: Sternorrhyncha for identification. - J. Trop. Agr. Res., 24(2): 139-149.

WiLLIAMs D.J., 1989 - The mealybug genus Rastrococcus Ferris (Hemiptera: Pseudococcidae). - Syst. Entomol., 14: 433-486.

Williams D.J., 2004 - Mealybugs of southern Asia. The Natural History Museum, London, U.K. Southdene SDN. BHD., Kuala Lumpur, 896 pp.

Williams D.J., Miller D.R., 2010 - Scale insects (Hemiptera: Sternorrhyncha: Coccoidea) of the Krakatau Islands including species from adjacent Java.Zootaxa, 2451: 43-52.

WIRJATI, 1958 - A small mealybug from a virgin forest. Idea, 31(11): 1. 
